\title{
Ablation of the pro-inflammatory master regulator miR-155 does not mitigate neuroinflammation or neurodegeneration in a vertebrate model of Gaucher's disease
}

\author{
Lisa Watson ${ }^{\mathrm{a}, \mathrm{b}}$, Marcus Keatinge ${ }^{\mathrm{a}, \mathrm{b}}$, Matthew Gegg ${ }^{\mathrm{c}}$, Qing Bai ${ }^{\mathrm{d}}, \mathrm{M}$. Cosmin Sandulescu ${ }^{\mathrm{d}}$, \\ Ayelet Vardi ${ }^{\mathrm{e}}$, Anthony H. Futerman ${ }^{\mathrm{e}}$, Anthony H.V. Schapira ${ }^{\mathrm{c}}$, Edward A. Burton ${ }^{\mathrm{d}}$, \\ Oliver Bandmann ${ }^{\mathrm{a}, \mathrm{b}, *}$ \\ ${ }^{a}$ The Bateson Centre, University of Sheffield, Sheffield, UK \\ ${ }^{\mathrm{b}}$ Sheffield Institute for Translational Neuroscience (SITraN), University of Sheffield, Sheffield, UK \\ ${ }^{\mathrm{c}}$ Department of Clinical and Movement Neurosciences, UCL Queen Square Institute of Neurology, London, UK \\ ${ }^{\mathrm{d}}$ Pittsburgh Institute for Neurodegenerative Diseases and Department of Neurology, University of Pittsburgh School of Medicine, Pittsburgh, USA \\ ${ }^{\mathrm{e}}$ Department of Biomolecular Sciences, Weizmann Institute of Science, Rehovot, Israel
}

\section{A R T I C L E I N F O}

\section{Keywords:}

Neuroinflammation

miR-155

Zebrafish

Gaucher's disease

Parkinson's disease

Neurodegeneration

\begin{abstract}
A B S T R A C T
Bi-allelic mutations in the glucocerebrosidase gene (GBA1) cause Gaucher's disease, the most common human lysosomal storage disease. We previously reported a marked increase in miR-155 transcript levels and early microglial activation in a zebrafish model of Gaucher's disease ( $\mathrm{gba1}^{-/-}$). miR-155 is a master regulator of inflammation and has been implicated in a wide range of different neurodegenerative disorders. The observed miR-155 upregulation preceded the subsequent development of widespread pathology with marked neuroinflammation, closely resembling human Gaucher's disease pathology. We now report similar increases of miR-155 expression in mammalian models of GD, confirming that miR-155 upregulation is a shared feature in glucocerebrosidase (GCase) deficiency across different species. Using CRISPR/Cas9 mutagenesis we then generated a miR-155 mutant zebrafish line (miR-155 ${ }^{-/-}$) with completely abolished miR-155 expression. Unexpectedly, loss of miR-155 did not mitigate either the reduced lifespan or the robust inflammatory phenotypes of $g b a 1^{-/-}$ mutant zebrafish. Our data demonstrate that neither neuroinflammation nor disease progression in GCase deficiency are dependent on miR-155 and suggest that miR-155 inhibition would not be a promising therapeutic target in Gaucher's disease.
\end{abstract}

\section{Introduction}

miR-155 is a typical multifunctional miRNA which is involved in a wide range of different immune and inflammatory processes (Faraoni et al., 2009). Inflammation is increasingly recognised as a key mechanism leading to neurodegenerative diseases with an observed upregulation of miR-155 in Parkinson's disease (PD), multiple sclerosis (MS), motor neuron disease (MND), Alzheimer's disease (AD) and other dementias (Butovsky et al., 2015; Caggiu et al. 2018; Chen et al., 2018; Tili et al. 2018). In MND, miR-155 is strongly upregulated in the spinal cord of both familial and sporadic MND with dysregulation of previously validated miR-155 downstream targets in the lumbar ventral horn (Butovsky et al., 2015). Genetic ablation of miR-155 ameliorated disease severity and prolonged survival in SOD1 $1^{\mathrm{G} 93 \mathrm{~A}}$ mice, a widely used rodent model of MND. Furthermore, treating SOD $1{ }^{\mathrm{G} 93 \mathrm{~A}}$ mice with anti-miR-155 at the beginning of disease onset also improved the disease phenotype and prolonged the lifespan, suggesting that miR-155 could be a therapeutic target for human MND (Butovsky et al., 2015).

In post-mortem studies of PD patient brains, activated microglia and increased concentrations of pro-inflammatory cytokines have been identified within the substantia nigra (Boka et al., 1994; McGeer et al., 1988). An increase in pro-inflammatory cytokines in both PD patient serum and cerebrospinal fluid (CSF) has been reported (Dobbs et al., 1999; Mogi et al., 1994). Distinct cytokine profiles may be associated with more aggressive progression of the disease (Williams-Gray et al., 2016). Genetic studies of risk factors for PD and epidemiological studies also support the role of inflammation in PD (Kaur et al. 2017). miR-155 is significantly upregulated in the substantia nigra pars compacta

\footnotetext{
* Corresponding author at: O. Bandmann, Sheffield Institute for Translational Neuroscience (SITraN), 385a Glossop Road, Sheffield S10 2HQ, UK.

E-mail address: o.bandmann@sheffield.ac.uk (O. Bandmann).
} 
A

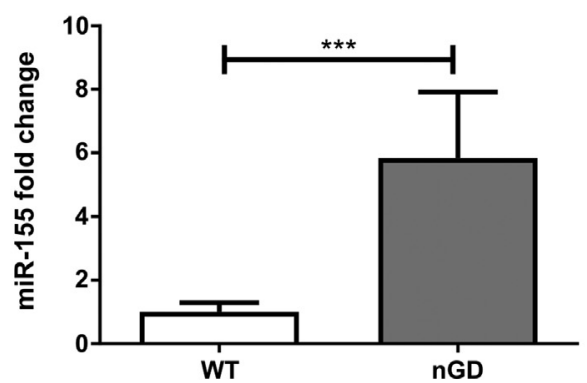

C

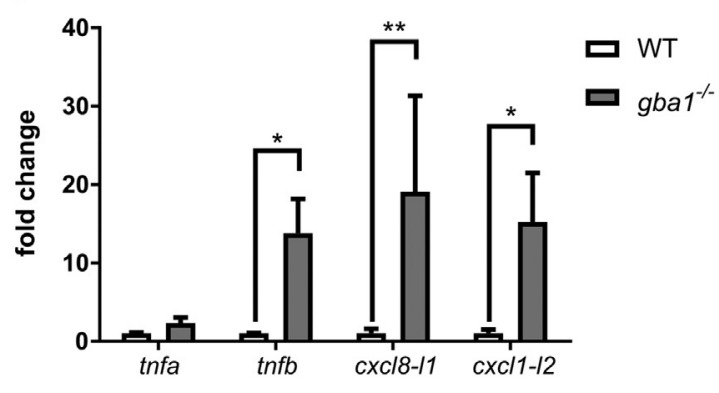

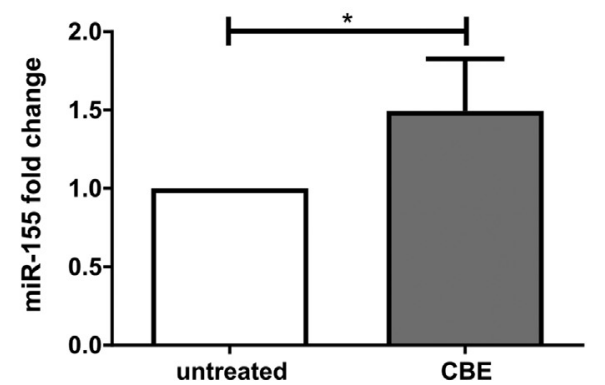

Fig. 1. miR-155 and associated inflammatory markers are upregulated in different models of GCase deficiency.

(A) miR-155 expression is upregulated in the cortex

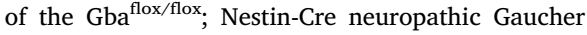
disease mouse model ( $n=6, p=.0004$, one-tailed $t$ test) and (B) in cultured murine microglia treated with the GCase inhibitor CBE ( $n=3, p=.031$, onetailed t-test). (C) Expression of miR-155 downstream targets tnfa, tnfb, cxcl8-l1 and cxcl8-l2 (zebrafish orthologues of human TNFa and IL-8) in 12 wpf zebrafish brain ( $\mathrm{n}=3$, two-way ANOVA). ${ }^{*} p<.05$, ** $p<.01,{ }^{* * *} p<.001$. Error bars $=$ SD.
(SNpc) of mice with adeno-associated-virus-mediated expression of alpha-synuclein. This rodent model of PD demonstrated microgliosis and a $30 \%$ loss of dopaminergic neurons. In contrast, $m i R-155^{-/-}$mice transduced with alpha-synuclein showed attenuated inflammatory responses and near complete neuroprotection (Thome et al., 2016).

Heterozygous mutations in GBA1 are the most common risk factor for PD (Gegg and Schapira, 2018) whilst bi-allelic GBA1 mutations cause Gaucher's disease (GD), the most common lysosomal storage disorder (Davidson et al., 2018). A key pathological feature of GD is the infiltration and accumulation of Gaucher cells in many different organs, particularly the haemopoietic system (Baris et al., 2014). Gaucher cells are infiltrating glycolipid-engorged macrophages and the source of increased inflammatory markers observed in GD patients (Panicker et al., 2014). Neuroinflammation is thought to be a major contributing feature to neurodegeneration in GD, as demonstrated by the upregulation of inflammatory markers, astrogliosis, and microglial activation correlating with neuronal loss in two murine models of GD (Farfel-Becker et al. 2011; Massaro et al., 2018; Vitner et al., 2012). Microglial activation is therefore a common feature in both PD and GD brains (Ginns et al., 2014). Of note, plasma levels of monocyte-associated inflammatory markers (including IL8, MCP-1, MIP1 $\alpha$, SCF, and PARC) are increased in $G B A 1^{+/-}$PD patients and increased IL8 levels were also associated with poorer cognition (Chahine et al., 2013). This suggests that inflammation may be a particularly important aspect of pathogenic mechanism in GBA-linked PD.

We recently described a $g b a 1$ mutant zebrafish line $\left(g b a^{-/-}\right)$generated by TALEN mutagenesis which faithfully modelled key aspects of human GD, including Gaucher cell accumulation, neurodegeneration and marked elevation of GD biomarkers. We observed early activation of immune/inflammatory mechanisms with microglial activation and increased miR-155 transcription levels at 4 or 5 days post fertilisation (dpf) respectively. Of note, both the early microglial activation and the accompanying miR-155 activation dramatically preceded the onset of a change in phenotype which only occurred at around 8-10 weeks with resulting lethality around 12 weeks of age. Compared to the larval stages, we observed a striking inflammatory phenotype at 12 weeks, with a further increase of miR-155 levels and marked microglial accumulation (Keatinge et al., 2015).

The aim of this study was therefore to investigate whether miR-155 upregulation may be a universal feature of GCase deficiency in mammalian models systems of GD and to determine whether miR-155 ablation may have a neuroprotective effect in gba1 deficiency.

\section{Methods}

\subsection{Zebrafish husbandry}

All larval and adult zebrafish were housed at the University of Sheffield; experimental procedures being in accordance UK Home Office Animals (Scientific Procedures) Act 1986 (Project licence PPL 70/8437, held by Professor Oliver Bandmann). Adult zebrafish were housed at a density of 40 per tank, on a cycle of $14 \mathrm{~h}$ of light, $10 \mathrm{~h}$ of dark. Adults and embryos were kept at constant temperature of $28^{\circ} \mathrm{C}$.

The $g b a 1^{-/-}$and pink $1^{-/-}$mutant lines were previously described in (Keatinge et al., 2015) and (Flinn et al., 2013) respectively. The miR155 mutant line was generated by the CRISPR/Cas9 method, using a gRNA targeting a Hyp188III site within the miR-155 coding region (ultramer sequence 5'-AAAGCACCGACTCGGTGCCACTTTTTCAAGTTG ATAACGGACTAGCCTTATTTTAACTTGCTATTTCTAGCTCTAAAACATC ACGATTAGCATTAAACCTATAGTGAGTCGTATTACGC-3'). Briefly, guide RNAs (gRNAs) were generated from a DNA ultramer template (IDT) and transcribed using a T7 MEGAshortscript ${ }^{\mathrm{TM}}$ kit (Invitrogen). gRNAs were then co-injected into one-cell stage zebrafish embryos with Cas9 protein (NEB). F0 injected embryos were raised to 3 months then crossed to wild type (WT) to identify founders. The progeny of selected F0 founders were then outcrossed to generate the F1 generation where appropriate mutant alleles were identified and selected for further study. The line was genotyped using primers F 5'-TATGACGTCCACAT GCATGC and R 5'-GATGTTCAGACGGTTCGCTC.

$\mathrm{miR}-155^{+/-}$were crossed to $\mathrm{gba1} 1^{+/-}$to generate double heterozygous individuals. These were subsequently in-crossed to generate double mutants. At each in-cross larvae were genotyped at $3 \mathrm{dpf}$ by larval tail biopsy as previously described (Wilkinson et al., 2013) and each genotype raised in genotype-specific tanks at a density of 10-15 fish per tank. All individuals were re-genotyped at 10 weeks post-fertilisation.

\subsection{Gene expression analyses}

RNA was extracted from zebrafish adult brains (12wpf), cortex of 21 day old $\mathrm{Gba}^{\text {flox/flox; }}$, nestin-Cre and $\mathrm{Gba}^{\text {flox/WT}}$; nestin-Cre mice, or BV2 microglial cells at specific time points using TRIzol ${ }^{\circledast}$ (Life 


\section{A dre-mir-155}

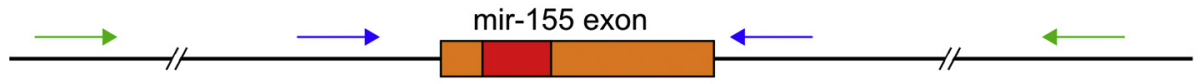

$\longrightarrow$ genotyping primers - WT product size: $148 \mathrm{~b}$

— mature miRNA sequence

whole region primers - WT product size: $1419 \mathrm{bp}$

dre-mir-155 mutation

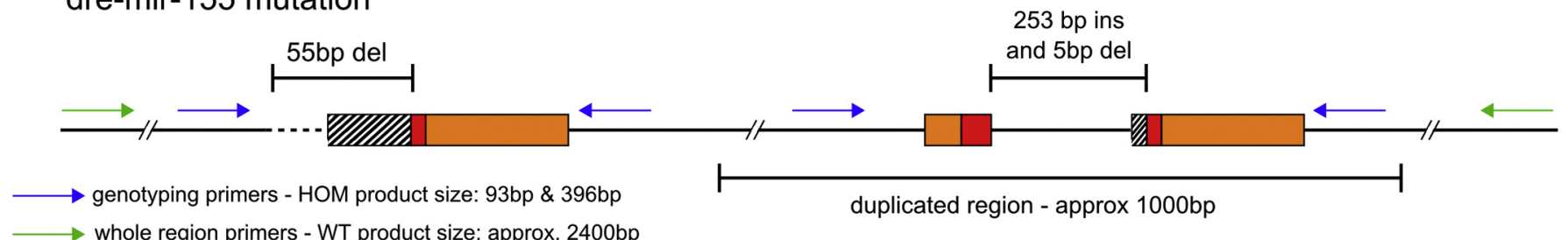

B

PCR with genotyping primers

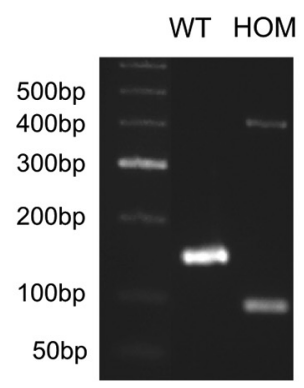

$\mathrm{D}$

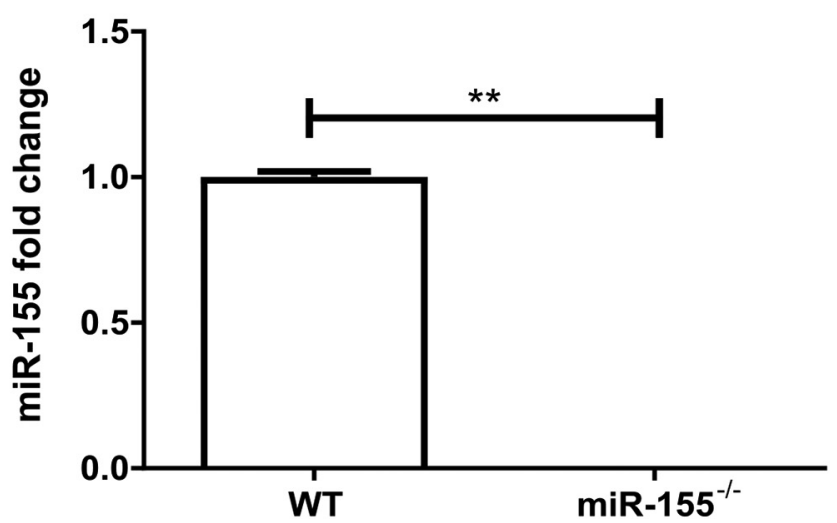

PCR with whole region primers

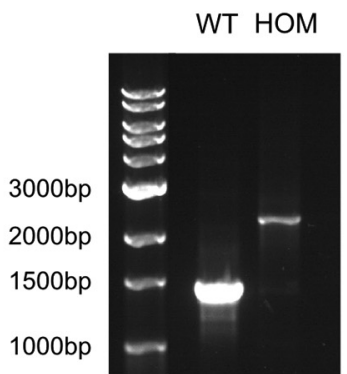

C

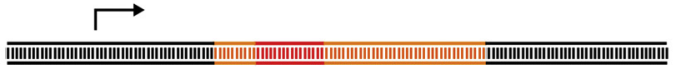

gene
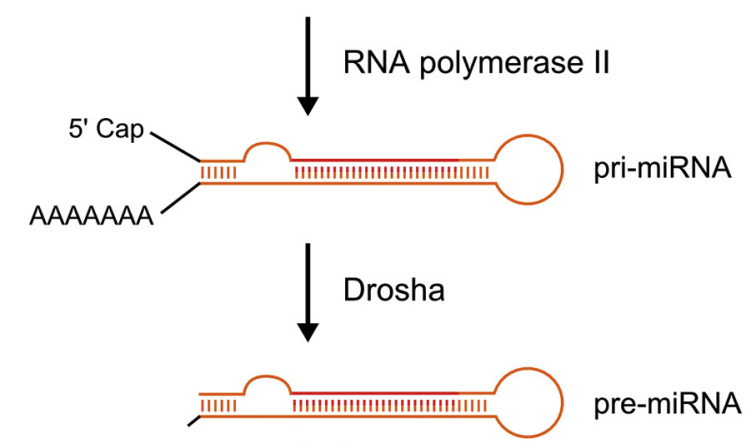

nucleus

cytosol
Dicer
IIIIIIIIIIIIIIIIIIIIII miRNA duplex

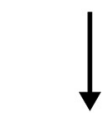

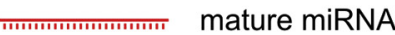

Fig. 2. Generation and validation of a miR-155 knockout line

CRISPR/Cas9 technology was used to generate a miR-155 knockout line. (A) This schematic shows the miR-155 gene of wild type (WT) mir-155 and the mutation generated by CRISPR/Cas9 mutagenesis. Briefly, in the mutant the whole mir-155 exon is duplicated (1000 bp duplicated region); in the first copy of mir-155 there is a $55 \mathrm{bp}$ deletion, and in the second copy there is a $253 \mathrm{bp}$ insertion and $5 \mathrm{bp}$ deletion. Both mutations disrupt the mature miR-155 sequence. (B) PCRs with genotyping and whole region primers confirm this complex mutation. In WT, only one amplicon of $148 \mathrm{bp}$, corresponding to the mir-155 exon, is generated using the genotyping primers (blue). In homozygous mutants (HOM), two amplicons are generated, one for each mir-155 exon. The amplicon spanning first exon generates a $93 \mathrm{bp}$ product reflecting the $55 \mathrm{bp}$ deletion. The amplicon spanning the second exon generates a $396 \mathrm{bp}$ product reflecting the $253 \mathrm{bp}$ insertion and $5 \mathrm{bp}$ deletion. The whole region primers (green) generate an amplicon of $1419 \mathrm{bp}$ in WT and an amplicon of approximately $2400 \mathrm{bp}$ in the HOMs reflecting the $1000 \mathrm{bp}$ insertion. The location of the PCR primers used to generate these products are shown in (A). (C) Schematic miRNA processing pathway, illustrating how the miR-155 mutation is predicted to result in abolished production of mature miR-155. (D) The predicted complete loss of mature miR-155 was validated by qPCR for miR-155 transcripts with miR-155 being undetectable in $\mathrm{miR}-155^{-/-}\left(\mathrm{n}=3, p=.0044\right.$, one-tailed $\mathrm{t}$-test). ${ }^{* *} p<.01$. (For interpretation of the references to colour in this figure legend, the reader is referred to the web version of this article.)

Technologies $^{\mathrm{TM}}$ ). cDNA was synthesised using a Verso ${ }^{\mathrm{TM}}$ reverse transcription kit (Thermo Fisher Scientific). Gene expression was determined using Brilliant II SYBR ${ }^{\circledR}$ Green QPCR Master Mix (Agilent Technologies) on a Stratagene MxPro 3000P (Stratagene) qPCR machine. For all experiments, ef1 $\alpha$ was used as a reference gene.
For miRNA qPCR analysis, RNA was extracted as above. For zebrafish and mouse brain RNA the RNA concentration was accurately quantified using the QuantiFluor ${ }^{\mathrm{TM}}$ RNA system (Promega) and the Qubit $^{\circledR}$ fluorometer (Life Technologies). 100 ng of total RNA was reverse-transcribed and subsequently qPCR was performed using TaqMan 
A

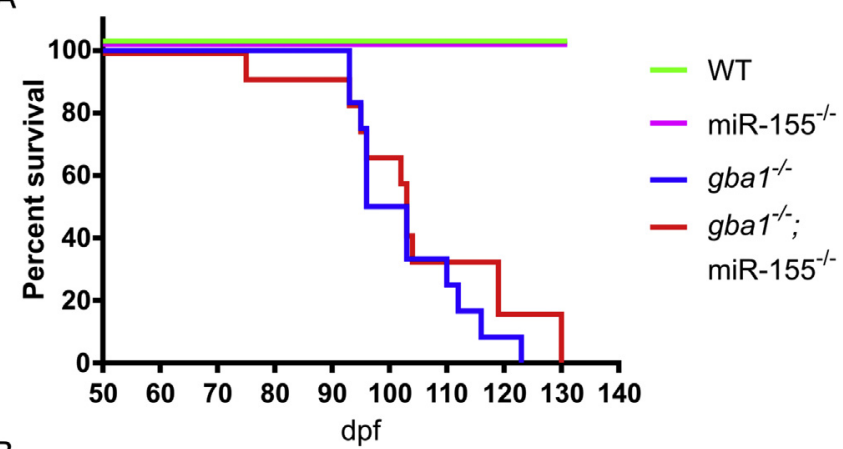

B

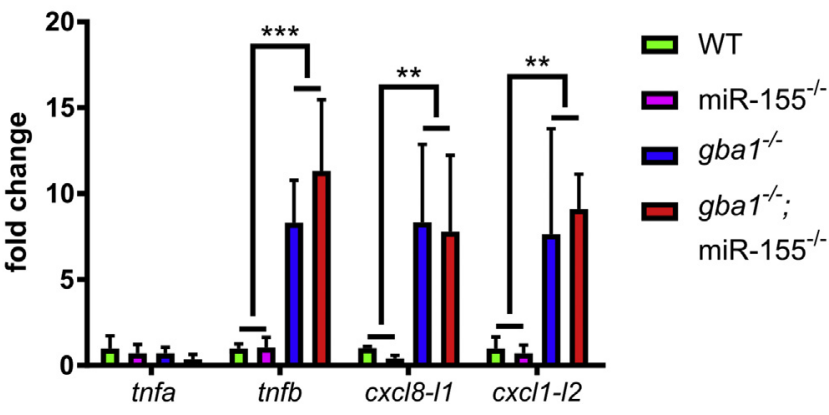

Fig. 3. Genetic ablation of miR-155 does not increase survival or ameliorate inflammatory phenotype in gba1 mutants.

(A) Lifespan of single ( $\left.\mathrm{gba}^{-/-}\right)$and double mutants $\left(\mathrm{gba1} 1^{-/-} ; \mathrm{miR}-155^{-/-}\right)$. The median survival was $99.5 \mathrm{dpf}$ in $\mathrm{gba1}^{-/-}$and $103 \mathrm{dpf}$ in $\mathrm{gba1}^{-/-}$;miR$155^{-/-}$( $n=11-13$ per group). (B) Loss of miR-155 does not affect expression of inflammatory markers as assessed by qPCR ( $n=4$, two-way ANOVA) * $\mathrm{p}<.05,{ }^{* *} \mathrm{p}<.01,{ }^{* * *}=\mathrm{p}<.001$. Error bars $=$ SD.

miRNA assays to quantify miR-155 levels. For mouse BV2 microglial cells miRNA was extracted from cells using PureLink miRNA isolation kit (ThermoFisher) and then converted to cDNA using TaqMan miRNA RT Kit (ThermoFisher). TaqMan miRNA assays were used to quantify miR-155 levels, and were normalised against U6 RNA (TaqMan, ThermoFisher).

All primer sequences listed in supplementary information.

\subsection{Microglial culture and CBE treatment}

Mouse BV2 microglial cells were grown in DMEM ( $4.5 \mathrm{~g} / \mathrm{L}$ glucose) supplemented with $10 \%(v / v)$ fetal calf serum treated with $100 \mu \mathrm{M} \mathrm{CBE}$ for 10 days, with the media changed every 3 days. miR-155 expression was then measured as stated above.

\subsection{IHC to assess microglial invasion of zebrafish brain}

Sample preparation for microglial IHC to investigate microglial activation in zebrafish brains was carried out as reported previously (Bai et al., 2014). Zebrafish were perfused and brains post-fixed in $4 \%$ PFA, followed by cryoprotection in PBS-sucrose. $14 \mu \mathrm{m}$-thick cryosections were mounted on glass slides, post-fixed, treated with $3 \% \mathrm{H}_{2} \mathrm{O}_{2}$ in PBS for $5 \mathrm{~min}$, then PBS-T (0.3\% Triton-X) for $1 \mathrm{~h}$, blocked with $10 \%$ goat serum in PBS for $2 \mathrm{~h}$ and then incubated overnight at $4{ }^{\circ} \mathrm{C}$ with primary antibody (7.4.C4, purified from hybridoma clone, \#92092321, HPA Culture Collections, UK), diluted 1:20 in PBS with 1\% goat serum. Primary antibody was detected using a biotinylated anti-mouse antibody (Vector Laboratories, Burlingame, CA, USA) followed by incubation with avidin-biotin-peroxidase complexes and staining using DAB (Vector Laboratories). Images were acquired using an Olympus BX51 compound light microscope. Microglia counts in multiple fields from 3 to 4 sections for 4 animals per group were made by an observer blinded to genotype.

\subsection{Statistical tests and analysis}

Graphpad prism V7 software (Graphpad) was used for statistical analysis and all errors bars shown denote standard deviation. All experiments were performed in biological triplicate unless otherwise stated. All data were analysed with either one-tailed $t$-test or two-way ANOVA with Tukey's post-tests.

\section{Results}

3.1. GCase deficiency leads to robust increase of miR-155 and downstream inflammatory markers in vitro and in vivo

To determine whether miR-155 was also upregulated in mammalian models of GCase deficiency, we assessed miR-155 expression in the

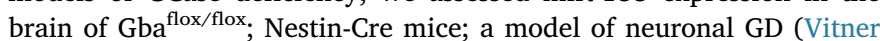
et al., 2012). In this model, miR-155 levels were 6-fold increased (Fig. $1 \mathrm{~A} ; n=6, p=.0004$, one-tailed t-test). To complement this data, we also analysed miR-155 expression in mouse BV2 microglial cells treated with the GCase inhibitor conduritol-B-epoxide (CBE) for 10 days. CBE treatment inhibited GCase activity by $90.6 \%$ (Control, $214.0 \pm 5.3 \mathrm{nmol} / \mathrm{h} / \mathrm{mg}$ protein; $\mathrm{CBE}, 20.1 \pm 3.2 \mathrm{nmol} / \mathrm{h} / \mathrm{mg}$ protein) and resulted in a 1.5-fold increase in miR-155 relative to untreated controls (Fig. 1B; $n=3, p=.031$, one-tailed t-test). These results confirm that miR-155 upregulation is not limited to $\mathrm{gba1}^{-/-}$zebrafish but can also be observed in mammalian models of GCase deficiency. To determine whether miR-155 levels were elevated in other zebrafish models of neurodegeneration, we analysed miR-155 levels in the brains of adult pink1 deficient zebrafish (Flinn et al., 2013). No increase in miR-155 was detected (data not shown).

To confirm a biological effect of increased miR-155 levels, we next determined the expression of its downstream target genes tnfa/tnfb (orthologous to human TNF $\alpha$ ) and cxcl8-l1/cxcl8-l2 (orthologous to human IL8) in $g b a 1^{-/-}$zebrafish brains (Lippai et al. 2013; Min et al., 2014; Pathak et al. 2015). Both IL8 orthologues (cxcl8-l1/cxcl8-l2) and tnfb were 15-20 fold upregulated in brain tissue from $g b a 1^{-/-}$zebrafish at $12 \mathrm{wpf}$ (Fig. 1C; $\mathrm{n}=3, p<.05$, two-way ANOVA). TNF $\alpha$ was also previously shown to be highly upregulated in brain tissue of $\mathrm{Gba}^{\text {flox/flox; }}$;estin-Cre mice (Vitner et al., 2012).

\subsection{Generation of a miR-155 knock out line using CRISPR/Cas9}

Having established that miR-155 upregulation is conserved in mammalian genetic and chemically-induced models of GCase deficiency, we next sought to determine whether miR-155 inactivation could be neuroprotective in the context of GCase deficiency. We used CRIPSR/Cas9 technology to generate a stable mutant miR-155 zebrafish line. A mutant with a complex genetic mutation was identified. There is a tandem duplication of the mir-155 gene, one copy carries a $55 \mathrm{bp}$ deletion and the other carries a large insertion (253 bp) and small $5 \mathrm{bp}$ deletion (Fig. 2A-B). miRNAs genes are transcribed by RNA polymerase II, the resultant transcript, usually around 80 nucleotides, adopts a hairpin loop secondary structure called the pri-miRNA. The pri-miRNA is exported to the cytoplasm and cleaved to produce the mature miRNA (Fig. 2C). The mutation generated in our mutant miR-155 zebrafish line significantly disrupts the sequence of the miRNA gene; we hypothesised that this would prevent the correct folding of the pri-miRNA and subsequent processing of the mature miRNA. Furthermore, the mature miRNA sequence itself is disrupted by the mutation. In the first miRNA gene resulting from the duplication only $5 \mathrm{bp}$ of the mature miRNA sequence remains; in the second, a large insertion is present in the middle of the mature miRNA sequence. To confirm the deleterious effect of the complex mir-155 mutation, a TaqMan ${ }^{\mathrm{TM}}$ MicroRNA Assay was applied. This assay uses a looped-primer RT-PCR to specifically recognise the mature miRNA sequence. As predicted, this experiment confirmed the complete absence of mature miR-155 in the brain tissue 
A

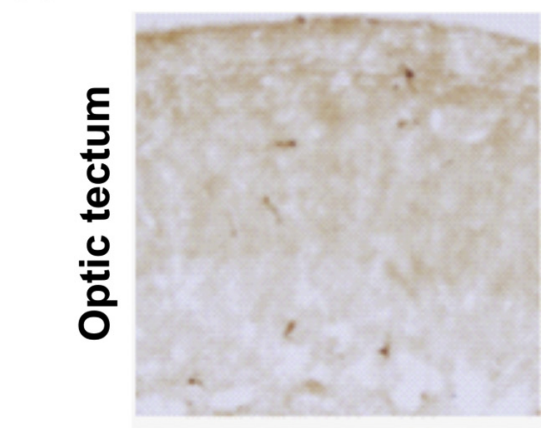

B

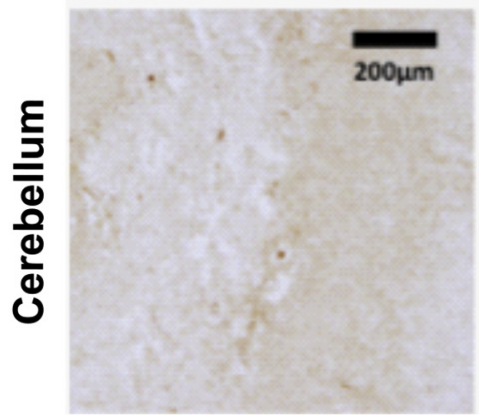

C

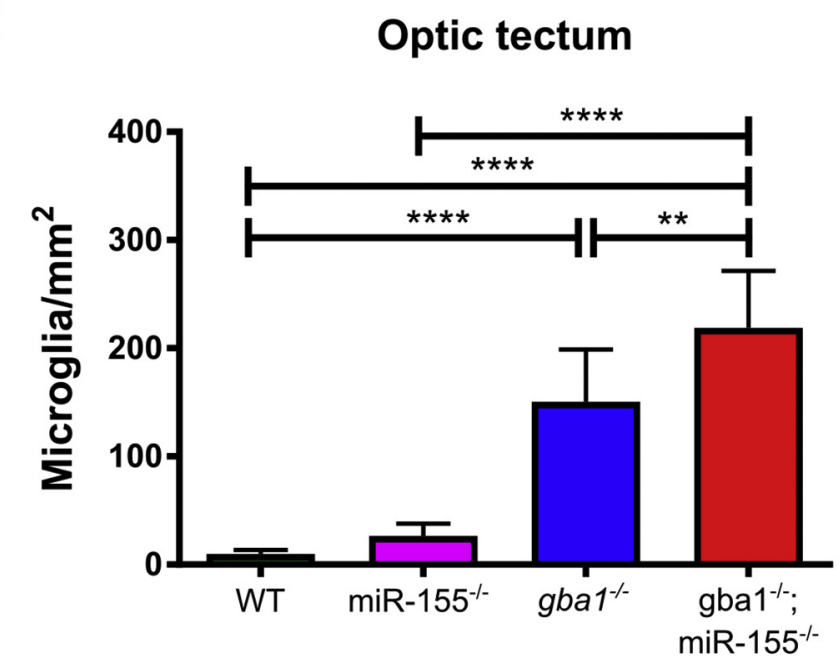

$\mathrm{miR}-155^{-/-}$
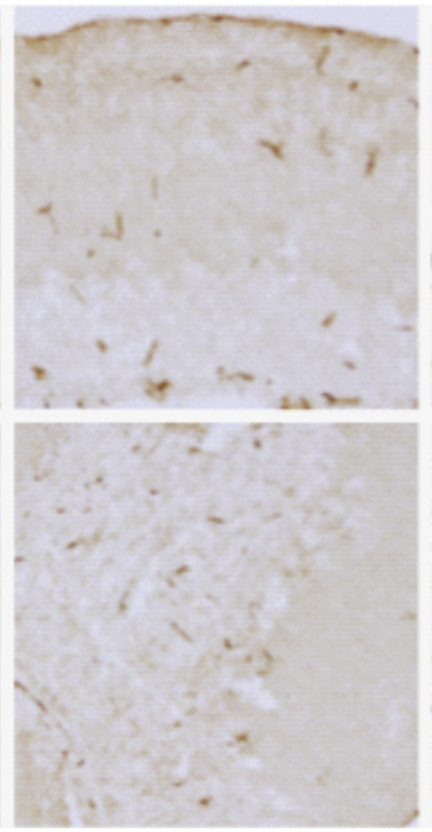

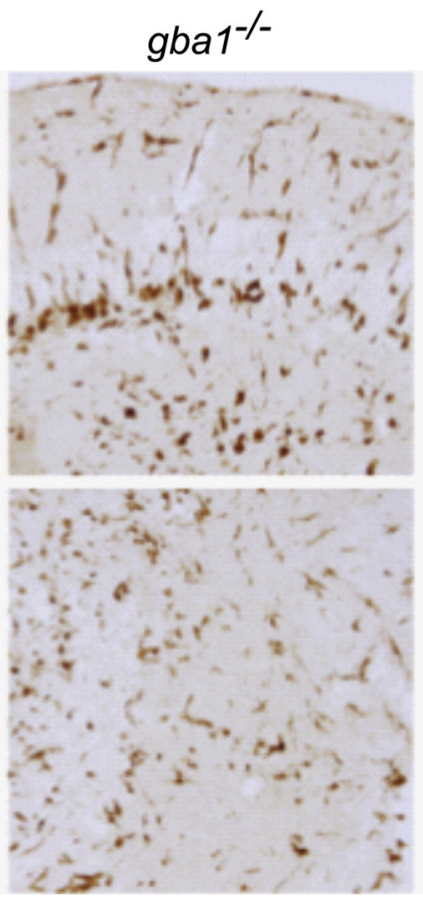

gba $1^{-/-}, \mathrm{miR}-155^{-/-}$

D

Cerebellum

Fig. 4. miR-155 ablation does not reduce microglial accumulation in $\mathrm{gba1}^{-/-}$zebrafish.

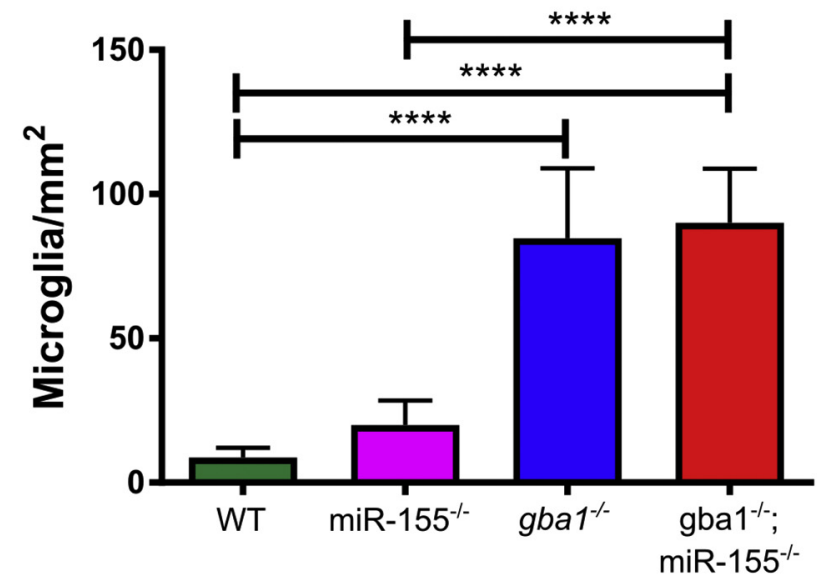

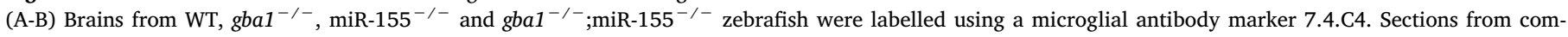
parable parts of the (A) optic tectum and (B) cerebellum are shown for each genotype, oriented rostral to left; scale bar for all images is shown in bottom left panel. (C-D) Microglial abundance was analysed for each genotype (2 sections each from 4 brains for each genotype). Immunoreactive microglia were quantified within each brain region of interest (C) optic tectum, (D) cerebellum and expressed as the density of microglia per square mm of brain region imaged ( $n=8$, two-way ANOVA). $* * \mathrm{p}<.01, * * * * p<.0001$. Error bars $=\mathrm{SD}$.

of miR-155 $5^{-1-}$ zebrafish (Fig. $2 \mathrm{D}, n=3, p=.0044$, one-tailed $t$-test).

\subsection{Genetic ablation of miR-155 does not increase survival or alter} inflammatory phenotypes of gba1 ${ }^{-/-}$zebrafish

To assess the effects of loss of miR-155 in $\mathrm{gba1}^{-/-}$zebrafish, miR$155^{+/-}$animals were crossed with $g b a 1^{+/-}$to generate $g b a 1^{+/-} ; \mathrm{miR}$ $155^{+/-}$zebrafish; these were subsequently in-crossed to generate double homozygous mutants ( $\left.\mathrm{gba1} 1^{-/-} ; \mathrm{miR}-155^{-/-}\right)$. Until 12 weeks post fertilisation, $g b a 1^{-/-}$zebrafish are visibly indistinguishable from their wild-type siblings. Around this age they develop a rapidly progressive movement phenotype and become emaciated. This is caused by extensive neurodegeneration accompanied by a strong neuroinflammatory phenotype (Keatinge et al., 2015). Unexpectedly, genetic ablation of miR-155 did not modulate the lifespan (Fig. 3A) of $\mathrm{gba}^{-/-}$zebrafish (median survival 99.5dpf in $\mathrm{gba1}^{-/-}$and 103dpf in gba1 $\left.{ }^{-/-} ; m i R-155^{-/-}, n=13\right)$ or the emergence of abnormal motor function (data not shown).

We next determined whether this absence of rescue in this model was attributable to lack of involvement of neuroinflammation in the phenotype versus miR-155 being unnecessary for neuroinflammation in this model. We had previously demonstrated marked microglial accumulation in the optic tectum and cerebellum in end stage $\mathrm{gba1}^{-/-}$ zebrafish (Keatinge et al., 2015). Genetic ablation of miR-155 did not ameliorate upregulation of tnfb or cxcl8-l1/l2 (Fig. 3B), or CNS microglial accumulation in $g b a 1^{-/-}$brains (Fig. 4). Unexpectedly, in the 
optic tectum, gba1 $1^{-/-} ;$miR- $155^{-/-}$zebrafish had a further $40 \%$ increase in microglial abundance compared to $\mathrm{gba1}^{-/-}$(Fig. 4C; 218 microglia/mm $\mathrm{mm}^{2}$ to $150 \mathrm{microglia} / \mathrm{mm}^{2}, n=8, p<.01$, two-way ANOVA with Tukey's post-tests).

\section{Discussion and conclusions}

There is growing interest in the role of epigenetic mechanisms in neurodegeneration but observations are frequently limited to model systems of one species and can be of uncertain functional relevance.

The mature sequence of miR-155 is $100 \%$ conserved between zebrafish and humans, similarly, the predicted binding sites in the 3'UTRs of many of the known targets of miR-155 are also conserved between zebrafish and humans. It is thought that miR-155 exerts its pro-inflammatory effects through targets such as SOCS1 which have key function as negative regulators on cytokines (Wang et al. 2018; Yao et al., 2012). Suppression of SOCS1, and other similar targets, by miR155 leads to an elevation of cytokine levels and pro-inflammatory effects (Testa et al., 2017). The miRNA target prediction software TargetScan predicts a miR-155 binding site in position 15-21 in the 3'UTR of zebrafish socs1.

We showed that miR-155 was upregulated in three vertebrate models of GCase deficiency: gba1 ${ }^{-/-}$zebrafish, Gba ${ }^{\text {flox/flox}}$; Nestin-Cre mice, and mouse BV2 microglia treated with CBE, a GCase inhibitor. We also showed that the orthologues of $T N F \alpha$ and $I L-8$, inflammatory markers which are regulated by direct targets of miR-155 (Lippai et al. 2013; Min et al., 2014; Pathak et al. 2015), are also highly upregulated in the brains of gba1 deficient zebrafish. IL- 8 was shown to be upregulated in PD patients with GBA mutations (Chahine et al., 2013) and TNF $\alpha$ is upregulated in GD patient serum and in the brain of $\mathrm{Gba}^{\text {flox/flox; }}$ Nestin-Cre mice (Barak et al., 1999; Vitner et al., 2012). These findings demonstrate that the pathogenic processes leading to miR-155 upregulation and inflammation is present in both mouse and zebrafish models of GCase deficiency.

We used CRISPR/Cas9 to generate a miR-155 mutant line and demonstrated with the Taqman ${ }^{\mathrm{TM}}$ miRNA assay that mature miR-155 is not produced in this miR-155 mutant line. In miR-155 mutants, the inflammatory marker cxcl8-l1, a classical miR-155 downstream target, was reduced $50 \%$ compared to WT zebrafish, however this did not reach significance. Unexpectedly, loss of miR-155 did not have an effect on the survival or inflammatory phenotypes of gba1 mutant zebrafish. This suggests that, at least in zebrafish, miR-155 does not play a causative role for neuroinflammation in GCase deficiency and suggests redundancy of inflammatory pathways. Loss of miR-155 had no effect on the upregulation of tnfb or $c x c x 8-l 1 / l 2$ in $g b a 1^{-/-}$zebrafish. This may be because of alternate pathways, which are not dependant on miR-155 targets, leading to their upregulation and the loss of repression of miR-155 targets may not be strong enough to overcome this effect. We cannot comment whether miR-155 is able to regulate socs 1 in zebrafish; luciferase assays or western blots are required to determine the effect of miRNAs on their direct targets as they exert their effects post-transcriptionally. It is possible that although the mature miR-155 sequence is conserved in zebrafish the binding sites in key anti-inflammatory targets may not be as well conserved and therefore miR155 may exert a weaker, or have no effect on these targets.

Although zebrafish are a powerful model for genetic studies and $70 \%$ of human genes have at least one clear orthologue, many genes have two orthologues due to a large genome duplication event in the zebrafish genome (Howe et al., 2013). In many cases it is not clear which gene copy is functional or, where both are, which is most closely related to its human counterpart. Our study provides evidence that the two zebrafish orthologues of $T N F \alpha$, tnfa and tnfb, function differently. We showed that in brains of 3-month-old gba1 mutant zebrafish tnfb was upregulated 8-fold while tnfa was unchanged, this highlights the importance of investigating both orthologues where they are present.

Our observation of marked pro-inflammatory changes in $\mathrm{gba1}^{-1}$
-'miR-155 ${ }^{-/-}$zebrafish suggests redundancy of pro-inflammatory pathways in GCase deficiency, at least in zebrafish this contrasts with a possible role of miR-155 in other forms of neurodegeneration. The observed rescue effect of miR-155 inactivation in mouse model systems of MND, PD and EAE may either reflect a more specific involvement of miR-155 in these conditions or reduced redundancy in mammalian model systems.

\section{Acknowledgements}

We gratefully acknowledge financial support from the Medical Research Council (MR/R011354/1) and Parkinson's UK (G1404) for OB, the National Institutes of Health (ES022644) and US Department of Veterans Affairs (BX003168) for EAB. The contents of this article do not represent the views of the US Department of Veterans Affairs or the United States Government.

\section{Appendix A. Supplementary data}

Supplementary data to this article can be found online at https:// doi.org/10.1016/j.nbd.2019.04.008.

\section{References}

Bai, Qing, Parris, Ritika S., Burton, Edward A., 2014. Different mechanisms regulate expression of Zebrafish myelin protein zero (P0) in myelinating oligodendrocytes and its induction following axonal injury. J. Biol. Chem. 289 (35), 24114-24128.

Barak, V., et al., 1999. Cytokines in Gaucher's disease. Eur. Cytokine Netw. 10 (2), 205-210.

Baris, Hagit N., Cohen, Ian J., Mistry, Pramod K., 2014. Gaucher disease: the metabolic defect, pathophysiology, phenotypes and natural history. Pediatr. Endocrinol. Rev. 12 (Suppl. 1), 72-81.

Boka, G., et al., 1994. Immunocytochemical analysis of tumor necrosis factor and its receptors in Parkinson's disease. Neurosci. Lett. 172 (1-2), 151-154.

Butovsky, Oleg, et al., 2015. Targeting miR-155 restores abnormal microglia and attenuates disease in SOD1 mice. Ann. Neurol. 77 (1), 75-99.

Caggiu, E., Paulus, K., Mameli, G., Arru, G., Sechi, G.P., Sechi, L.A., 2018 Sep 14. Differential expression of miRNA 155 and miRNA 146a in Parkinson's disease patients. eNeurologicalSci. 13, 1-4. 10.1016/j.ensci.2018.09.002.

Chahine, Lama M., et al., 2013. Clinical and biochemical differences in patients having Parkinson disease with vs without GBA mutations. JAMA Neurol. 70 (7), 852.

Chen, Jing, et al., 2018 Jun. MicroRNA expression data analysis to identify keymiRNAs associated with Alzheimer's disease. J. Gene Med. 20 (6), e3014.

Davidson, Brad A., et al., 2018. Exploring genetic modifiers of Gaucher disease: the next horizon. Hum. Mutat. 1-13 May.

Dobbs, R.J., et al., 1999. Association of circulating TNF-alpha and IL-6 with ageing and parkinsonism. Acta Neurol. Scand. 100 (1), 34-41.

Faraoni, Isabella, Antonetti, Francesca Romana, Cardone, John, Bonmassar, Enzo, 2009 miR-155 gene: a typical multifunctional microRNA. Biochim. Biophys. Acta Mol. basis Dis. 1792 (6), 497-505.

Farfel-Becker, T., Vitner, E.B., Pressey, S.N., Eilam, R., Cooper, J.D., Futerman, A.H., 2011 Apr 1. Spatial and temporal correlation between neuron loss and neuroinflammation in a mouse model of neuronopathic Gaucher disease. Hum Mol Genet 20 (7), 1375-1386.

Flinn, Laura J., et al., 2013. TigarB causes mitochondrial dysfunction and neuronal loss in PINK1 deficiency. Ann. Neurol. 74 (6), 837-847.

Gegg, Matthew E., Schapira, Anthony H.V., 2018. The role of glucocerebrosidase in Parkinson disease pathogenesis. FEBS J. 1-13.

Ginns, Edward I., et al., 2014. Neuroinflammation and $\alpha$-Synuclein accumulation in response to glucocerebrosidase deficiency are accompanied by synaptic dysfunction. Mol. Genet. Metab. 111 (2), 152-162.

Howe, Kerstin, et al., 2013. The Zebrafish reference genome sequence and its relationship to the human genome. Nature 496 (7446), 498-503.

Kaur, K., Gill, J.S., Bansal, P.K., Deshmukh, R., 2017 Oct 15. Neuroinflammation - A major cause for striatal dopaminergic degeneration in Parkinson's disease. J Neurol Sci 381, 308-314. 10.1016/j.jns.2017.08.3251.

Keatinge, Marcus, et al., 2015. Glucocerebrosidase 1 deficient Danio Rerio Mirror key pathological aspects of human Gaucher disease and provide evidence of early microglial activation preceding alpha-Synuclein-independent neuronal cell death. Hum. Mol. Genet. 24 (23), 6640-6652.

Lippai, D., Bala, S., Csak, T., Kurt-Jones, E.A., Szabo, G., 2013 Aug 9. Chronic alcoholinduced microRNA-155 contributes to neuroinflammation in a TLR4-dependent manner in mice. PLoS One 8 (8). 10.1371/journal.pone.0070945.

Massaro, Giulia, et al., 2018. Fetal gene therapy for neurodegenerative disease of infants. Nat. Med. 24 (9), 1317-1323.

McGeer, P.L., Itagaki, S., Boyes, B.E., McGeer, E.G., 1988. Reactive microglia are positive for HLA-DR in the Substantia Nigra of Parkinson's and Alzheimer's disease brains. Neurology 38 (8), 1285-1291. 
Min, Min, et al., 2014. MicroRNA-155 is involved in the pathogenesis of ulcerative colitis by targeting FOXO3a. Inflamm. Bowel Dis. 20 (4), 652-659.

Mogi, M., et al., 1994. Tumor necrosis factor-alpha (TNF-alpha) increases both in the brain and in the cerebrospinal fluid from Parkinsonian patients. Neurosci. Lett. 165 (1-2), 208-210.

Panicker, Leelamma M., et al., 2014. Gaucher iPSC-derived macrophages produce elevated levels of inflammatory mediators and serve as a new platform for therapeutic development. Stem cells (Dayton, Ohio) 32 (9), 2338-2349.

Pathak, S., Grillo, A.R., Scarpa, M., Brun, P., D'Incà, R., Nai, L., Banerjee, A., Cavallo, D., Barzon, L., Palù, G., Sturniolo, G.C., Buda, A., Castagliuolo, I., 2015 May 22. MiR-155 modulates the inflammatory phenotype of intestinal myofibroblasts by targeting SOCS1 in ulcerative colitis. Exp Mol Med 47, e164. 10.1038/emm.2015.21.

Testa, Ugo, Pelosi, Elvira, Castelli, Germana, Labbaye, Catherine, 2017. miR-146 and miR-155: two key modulators of immune response and tumor development. NonCoding RNA 3 (3), 22.

Thome, A.D., Harms, A.S., Volpicelli-Daley, L.A., Standaert, D.G., 2016. MicroRNA-155 regulates alpha-synuclein-induced inflammatory responses in models of Parkinson disease. J. Neurosci. 36 (8), 2383-2390.

Vitner, Einat B., et al., 2012. Contribution of brain inflammation to neuronal cell death in Neuronopathic forms of Gaucher's disease. Brain 135 (6), 1724-1735.

Wang, D., Tang, M., Zong, P., Liu, H., Zhang, T., Liu, Y., Zhao, Y., 2018 Jun 8. MiRNA-155 Regulates the Th17/Treg Ratio by Targeting SOCS1 in Severe Acute Pancreatitis. Front Physiol 9. 10.3389/fphys.2018.00686.

Wilkinson, Robert N., Elworthy, Stone, Ingham, Philip W., van Eeden, Fredericus J.M., 2013. A method for high-throughput PCR-based genotyping of larval Zebrafish tail biopsies. BioTechniques 55 (6), 314-316.

Williams-Gray, Caroline H., et al., 2016. Serum immune markers and disease progression in an incident Parkinson's disease cohort (ICICLE-PD). Mov. Disord. 31 (7), 995-1003.

Yao, Rui, et al., 2012. MicroRNA-155 modulates Treg and Th17 cells differentiation and Th17 cell function by targeting SOCS1. PLoS One 7 (10), e46082. 